\title{
Universal Scaling in the Dynamic Hysteresis, and Non-Markovian Dynamics, of a Tunable Optical Cavity
}

\author{
Z. Geng, ${ }^{1}$ K. J. H. Peters $\odot,{ }^{1}$ A. A. P. Trichet $\odot,{ }^{2}$ K. Malmir $\odot,{ }^{2}$ R. Kolkowski, ${ }^{1}$ J. M. Smith, ${ }^{2}$ and S. R. K. Rodriguez $\oplus^{1,}{ }^{*}$ \\ ${ }^{1}$ Center for Nanophotonics, AMOLF, Science Park 104, 1098 XG Amsterdam, Netherlands \\ ${ }^{2}$ Department of Materials, University of Oxford, Parks Road, Oxford OX1 3PH, United Kingdom
}

(Received 1 November 2019; accepted 31 March 2020; published 15 April 2020)

\begin{abstract}
We investigate, experimentally and theoretically, the dynamics of a laser-driven cavity with noninstantaneous effective photon-photon interactions. Scanning the laser-cavity frequency detuning at different speeds across an optical bistability, we find a hysteresis area that is a nonmonotonic function of the speed. In the limit of fast scans comparable to the memory time of the interactions, we demonstrate that the hysteresis area decays following a universal power law with scaling exponent -1 . We further demonstrate a regime of non-Markovian dynamics emerging from white noise. This regime is evidenced by peaked distributions of residence times in the metastable states of our system. Our results offer new perspectives for exploring the physics of scaling, universality, and metastability, in non-Markovian regimes using arrays of bistable optical cavities with low quality factors, driven by low laser powers, and at room temperature.
\end{abstract}

DOI: 10.1103/PhysRevLett.124.153603

Photons in a nonlinear cavity can undergo phase transitions akin to condensed matter systems. Since the seminal works by Graham and Haken [1], Roy and Mandel [2], and Scully [3], lasers have inspired numerous studies of phase transitions of light. Recently, coherently driven cavities supporting mean-field bistability — two steady states at a single driving condition-have taken a central role in studies of photonic phase transitions [4-17]. Progress in this field has been recently accelerated by three developments. First, various nonlinear photonic resonators, and novel methods to probe their dynamics, are becoming available $[8,9,13-15]$. Second, fresh insights coupled to novel theoretical methods have revealed intriguing nonequilibrium phases of nonlinear cavities [4,7,10,12,18-21]. Third, there is increasing interest in performing optimization [22-24] and computation [25] with bistable cavity arrays.

Descriptions of bistable cavities commonly assume instantaneous effective photon-photon interactions [26]. In the mean-field equation of motion for the intracavity field $\alpha$, this assumption manifests as a Kerr nonlinearity of the form $|\alpha|^{2} \alpha$ [27]. The same cubic nonlinearity is found in the Gross-Pitaevskii equation employed in atomic physics [28-30], in the Ginzburg-Landau theory of superconductivity [31], in the Lugiato-Lefever equation of nonlinear optics [32], and in the force derived from Goldstone's

Published by the American Physical Society under the terms of the Creative Commons Attribution 4.0 International license. Further distribution of this work must maintain attribution to the author(s) and the published article's title, journal citation, and DOI.
Mexican hat potential $V=-|\phi|^{2}+|\phi|^{4}$ for the scalar field $\phi$ at the heart of the Higgs mechanism [33]. In optics, strong Kerr nonlinearities arise in semiconductor cavities where exciton-exciton interactions are effectively instantaneous [26]. A drawback of those cavities is that optical bistability based on Kerr nonlinearities is typically observed only at cryogenic temperatures. In contrast, several optical resonators with slow but strong thermal nonlinearities have routinely displayed bistability at room temperature [34-40]. As bona fide bistable systems, thermo-optical resonators may open up new perspectives for classical Hamiltonian simulation and computation [22-25,41]. However, the influence of the thermal relaxation time on the hysteretic and stochastic dynamics of bistable cavities remains to be addressed.

In this Letter, we demonstrate signatures of scaling, universality, and non-Markovianity, in the dynamics of a laser-driven cavity with thermo-optical nonlinearity. This nonlinearity is associated with noninstantaneous effective photon-photon interactions, and it results in room-temperature optical bistability at low laser powers $P \sim 70 \mu \mathrm{W}$. Scanning the laser-cavity detuning, we observe an optical hysteresis influenced by the ratio of the scanning time to the memory time of the interactions. In contrast to previous reports of dynamic hysteresis in resonators with effectively instantaneous interactions [13,42-44], we find a hysteresis area that is a nonmonotonic function of the scanning speed. Moreover, we discover a universal scaling of the hysteresis area in the limit of fast scans. Our results elucidate how the hysteretic behavior characterizing first-order phase transitions, and the boundary between phases, dynamically vanish when the nonlinearity has a finite memory time. Furthermore, we evidence a new regime of non-Markovian 
dynamics characterized by peaked distributions of residence times in metastable states. Interestingly, this new non-Markovian regime emerges under the influence of white noise.

The inset in Fig. 1 illustrates our system: a tunable Fabry-Pérot cavity driven by a $532 \mathrm{~nm}$ continuous wave laser. The cavity is made by a concave and a planar mirror, each comprising a distributed Bragg reflector (DBR) on a glass substrate. The mirrors have a peak reflectance of $99.9 \%$ at $530 \mathrm{~nm}$, which is the center of the stop band. The concave mirror, with a diameter of $7 \mu \mathrm{m}$ and a radius of curvature of $12 \mu \mathrm{m}$, was fabricated by milling a glass substrate with a focused-ion beam prior to the deposition of the DBR [45]. An image of the concave mirror is shown in Supplemental Material [46]. Single-mode operation is ensured by the strong lateral confinement and high reflectivity of our mirrors.

The mirrors are aligned parallel to each other by controlling all three translational (rotational) degrees of freedom of the concave mirror with nanometer (microdegree) precision using a nanopositioner. The planar mirror is mounted on another actuator used to scan the cavity length. Optical excitation and collection are achieved through $10 \times$ microscope objectives with numerical aperture NA $=0.25$. The cavity transmission is measured by a photodetector and an oscilloscope. Setup details are in Supplemental Material [46].

To endow the cavity with a nonlinear optical response, we place olive oil inside [47-49]. Through $z$-scan measurements, we estimated the nonlinear refractive index $n_{2}$ of our oil to be $\sim-5 \times 10^{-8} \mathrm{~cm}^{2} / \mathrm{W}$ at $532 \mathrm{~nm}$, consistent with Ref. [49]. Figure 1 shows the transmitted intensity

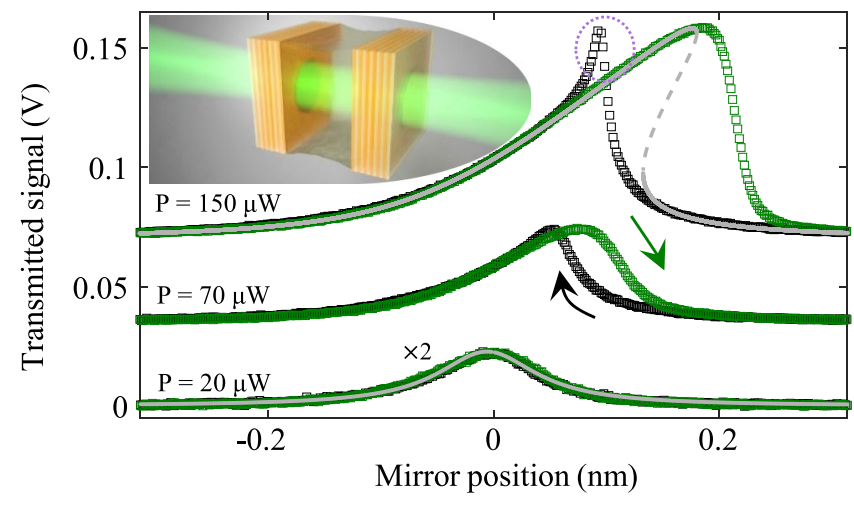

FIG. 1. Inset: Schematic of a planar-concave microcavity filled with oil, as in our experiments. Main: Average hysteresis measured by scanning $\Delta / \Gamma$ [see Eq. (1)] at constant speed for three driving powers $P$. Green (black) curves correspond to opening (closing) the cavity. The overshoot enclosed by the dotted circle emerges from noninstantaneous effective photonphoton interactions. For clarity, we multiplied the measurements for $P=20 \mu \mathrm{W}$ by 2 and vertically displaced the other measurements. Gray curves are calculations with Eq. (1) as explained in the text. through our oil-filled cavity averaged over 70 cycles and at three laser powers. Green and black data points correspond to opening and closing the cavity, respectively. For low powers $P \lesssim 20 \mu \mathrm{W}$, the cavity response is linear. The gray curve over the measurements for $P=20 \mu \mathrm{W}$ is a Lorentzian fit, yielding a resonance linewidth of $0.104 \pm 0.001 \mathrm{~nm}$. For $P=70 \mu \mathrm{W}$, the transmission displays hysteresis (see arrows in Fig. 1) and bistability around a mirror position of $0.1 \mathrm{~nm}$. The power needed for bistability in our cavity is similar to that in state-of-theart monolithic semiconductor cavities $[13,15,50]$ but at conveniently lower quality factors (by a factor of $\sim 10$ ) and operating at room temperature instead of $\sim 5 \mathrm{~K}$. For $P=150 \mu \mathrm{W}$, the bistability and hysteresis range enlarge as expected. In Supplemental Material, we estimate the temperature rise in our cavity [46], based on the refractive index of olive oil as a function of the temperature [51].

All measurements in Fig. 1 correspond to linear ramps of the cavity length at $1.75 \mu \mathrm{m} / \mathrm{s}$. Already for this slow scan, an overshoot followed by a slow decay of the transmitted intensity arises when closing the cavity in the nonlinear regime. This overshoot is due to the finite thermal relaxation time of the oil-filled cavity, which is absent in the standard Kerr nonlinearity [35].

The standard Kerr model for the intracavity mean-field $\alpha$ in a frame rotating at the driving frequency $\omega$ is

$$
i \dot{\alpha}=\left(-\Delta-i \frac{\Gamma}{2}+U\left(|\alpha|^{2}-1\right)\right) \alpha+i \sqrt{\kappa_{1}} F .
$$

$\Delta=\omega-\omega_{0}$ is the laser-cavity detuning, with $\omega_{0}$ the resonance frequency. $U$ is the effective photon-photon interaction strength mediated by the nonlinear medium [26]. $F$ is the driving amplitude. The total loss rate $\Gamma=$ $\kappa_{1}+\kappa_{2}+\gamma$ is the sum of the input-output leakage rates through the two mirrors, $\kappa_{1,2}$, and the intrinsic cavity loss rate $\gamma$ due to absorption. The steady states follow from setting $\dot{\alpha}=0$ in Eq. (1).

We attempted to fit the steady-state photon density $|\alpha|^{2}$ calculated with Eq. (1) to the measurements for $P=$ $150 \mu \mathrm{W}$ in Fig. 1, with $F$ as the only relevant adjustable parameter [46]. $\Gamma$ is fixed by the measured linewidth in the linear regime. Furthermore, since Eq. (1) is a mean-field model, the absolute values of $|\alpha|^{2}$ and $U$ are irrelevant; the spectral line shape is determined by the ratio $U|\alpha|^{2} / \Gamma$. Various line shapes can be obtained by varying $F$ for fixed $U$ and $\Gamma$. Thus, we adjusted $F$ until obtaining the gray curve plotted over the measurements for $P=150 \mu \mathrm{W}$. Solid and dashed curves represent stable and unstable states, respectively. Stability was analyzed as in Refs. [27,52]. The fit is good far from resonance but deviates from the data near the bistability. This deviation increases with the scanning speed, as shown next.

We performed hysteresis measurements for $P=$ $150 \mu \mathrm{W}$ and various scanning speeds. A laser power far 
above the bistability threshold limits the influence of noise on our measurements. Figure 2(a) shows average hysteresis measurements for three speeds. Top to bottom, the speed is $\nu, 7 \nu$, and $49 \nu$, with $\nu=0.74 \mu \mathrm{m} / \mathrm{s}$. The transmitted intensity is shown versus $\Delta / \Gamma$, determined by the mirror position and resonance linewidth in Fig. 1. Figure 2(a) shows how the hysteresis cycle changes with the scanning speed. Increasing the speed from $\nu$ to $7 \nu$ makes the overshoot broader and the hysteresis wider. Interestingly, further increasing the speed to $49 \nu$ makes the overshoot broader but the hysteresis narrower. At $49 \nu$, the line shape for both scanning directions resembles a Lorentzian, which suggests approximately a linear response for fast scans regardless of the power.

The behavior in Fig. 2(a) can be explained by considering the finite heating and cooling time of our oil-filled microcavity; this makes effective photon-photon interactions noninstantaneous. Therefore, we modify Eq. (1) by letting

$U\left[|\alpha(t)|^{2}-1\right] \rightarrow \int_{0}^{t} d s K(t-s)\left[|\alpha(s)|^{2}-1\right] \equiv w(t)$,

with the kernel function defined as $K(t)=(U / \tau) e^{-t / \tau}$. The memory time of the interactions, $\tau$, corresponds to the thermal relaxation time of our microcavity. Here, we have followed the prescription of Mori [53] and Hänggi [54] for dealing with finite-time interactions. However, whereas
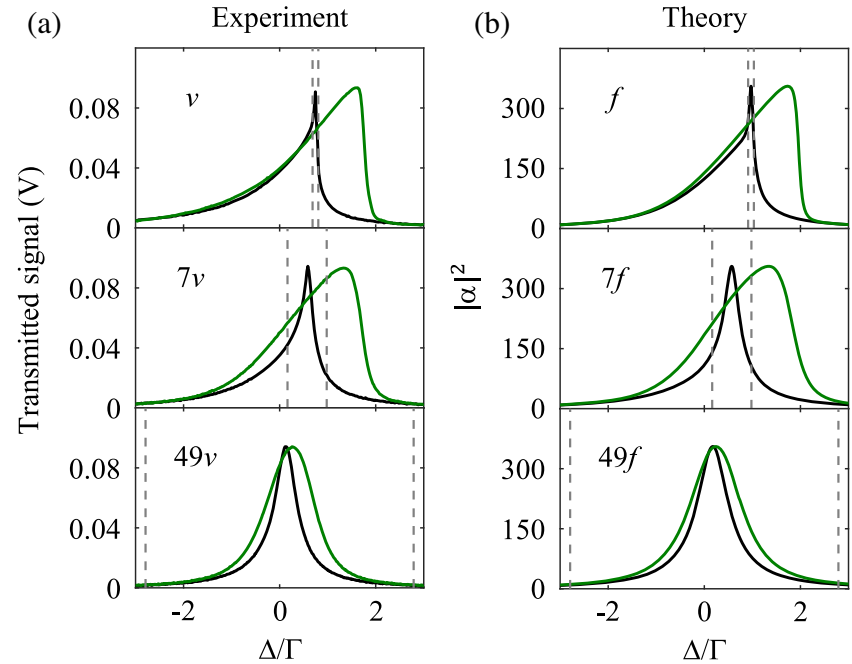

FIG. 2. (a) Measurements and (b) calculations of average hysteresis when $\Delta / \Gamma$ is scanned at three different speeds and constant power. The slowest scanning speed is $\nu=0.74 \mu \mathrm{m} / \mathrm{s}$ in (a) and $f=5.97 \times 10^{-6} \Gamma^{2}$ in (b). The power is $P=150 \mu \mathrm{W}$ in (a) and $F=1.52 F_{c}$, with $F_{c}$ the critical amplitude needed for bistability, in (b); the power in (a) also corresponds to $F=1.52 F_{c}$. Measurements are averaged over 70 realizations. Dashed lines indicate the range of $\Delta / \Gamma$ corresponding to the thermal relaxation time $\tau$.
Mori-type equations involve noninstantaneous dissipation, we introduced noninstantaneous nonlinearity.

Making the substitution (2) in Eq. (1) yields an integrodifferential equation, which can be conveniently (for numerical simulation) written as two coupled differential equations:

$$
\begin{gathered}
i \dot{\alpha}(t)=\left(-\Delta-i \frac{\Gamma}{2}+w(t)\right) \alpha(t)+i \sqrt{\kappa_{1}} F, \\
\dot{w}(t)=\left\{U\left[|\alpha(t)|^{2}-1\right]-w(t)\right\} / \tau .
\end{gathered}
$$

Equations (2) and (3) imply that the state of the system depends on its entire past, weighted by the memory kernel $K(t)$. Thus, interactions are nonlocal in time. Note that when $\alpha(t)$ is constant and can be taken out of the integral in Eq. (2), we recover Eq. (1). Hence, steady states are unchanged by $K(t)$.

Figure 2(b) shows dynamic hysteresis calculations using Eqs. (3), with the same parameter values used for the steady-state calculations in Fig. 1. As for the experiments, we show scanning speeds a factor of 7 apart. The model reproduces all features observed in experiments. In the calculations, we set the memory time to $\tau=10^{4} \Gamma^{-1}$ and the slowest scanning speed to $f=5.97 \times 10^{-6} \Gamma^{2} \quad$ [46]. Relative to the experiments, the value of $\tau$ is smaller (details ahead) and the speed is larger. We rescaled timescales to avoid unnecessarily long and memory-expensive calculations. Our mean-field calculations can be directly compared to experiments, because we respect the hierarchy of timescales in experiments: $\Gamma^{-1} \ll \tau \lesssim T_{b}$, with $T_{b}$ the scanning time across the bistability. Moreover,the ratio $T_{b} / \tau$ is similar for experiments and calculations.

In Fig. 3(a), we plot the experimental average hysteresis area $A=\int_{0}^{T}\left|I_{\Delta \uparrow}-I_{\Delta \downarrow}\right| d t$, with $I_{\Delta \uparrow}$ and $I_{\Delta \downarrow}$ the transmitted intensity when $\Delta$ increases and decreases, respectively. $T$ is the driving period, which exceeds $T_{b}$. Figure 3(b) shows the corresponding calculations based on Eqs. (3). In both measurements and calculations, $A$ peaks at a scanning speed
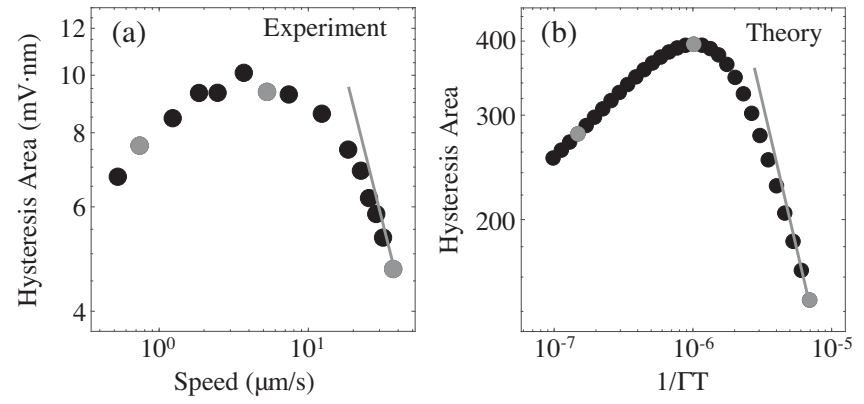

FIG. 3. (a) Measured and (b) calculated hysteresis area versus scanning speed, in the same dynamic range. Measurements are averaged over 70 realizations. Gray data points indicate the speeds presented in Fig. 2. Gray lines are power laws with exponent -1 . 
corresponding to the crossover between two regimes. For slow scans, $A$ increases with the speed, because the cavity cannot adiabatically follow $\Delta(t)$. This regime of dynamic hysteresis and the corresponding scaling laws for $A$ have been previously explored $[13,42,43,55]$. The second and new regime we investigate comprises speeds above the value for which $A$ peaks. Therein, $A$ decays with increasing speed, because the nonlinearity does not have time to build up during the scan. At high speeds, the dynamics of the system transition from nonlinear to linear. The number of attractors changes from two to one.

Our measurements are limited to scanning speeds between $\sim 0.5$ and $\sim 40 \mu \mathrm{m} / \mathrm{s}$. The upper speed limit is determined by the resonance frequency of our piezoelectric actuator. On the other end, we limited our measurements to speeds above $0.5 \mu \mathrm{m} / \mathrm{s}$ to avoid low-frequency mechanical noise in our setup. Despite these limitations, the good agreement between experiments and calculations encourages us to use our model to interpret the physics over an extended speed range.

In Fig. 4, we calculate $A$ versus the scanning speed for different $F$. At low speeds, the driving conditions determine the scaling of $A$ [13]. At high speeds, $A$ decays following a power law with scaling exponent -1 . Interestingly, this scaling behavior is universal; i.e., the slope of the gray lines fitted to the data in Fig. 4 is independent of the system parameters. To assess whether our experiments display such scaling behavior, in Fig. 3(a) we plot a power law with exponent -1 over our high-speed data points. The fit suggests that we reached the onset of the -1 power law regime. For comparison, we plot a -1 power law on top of the corresponding calculations in Fig. 3(b). In this case, the power law was fitted to the calculations in

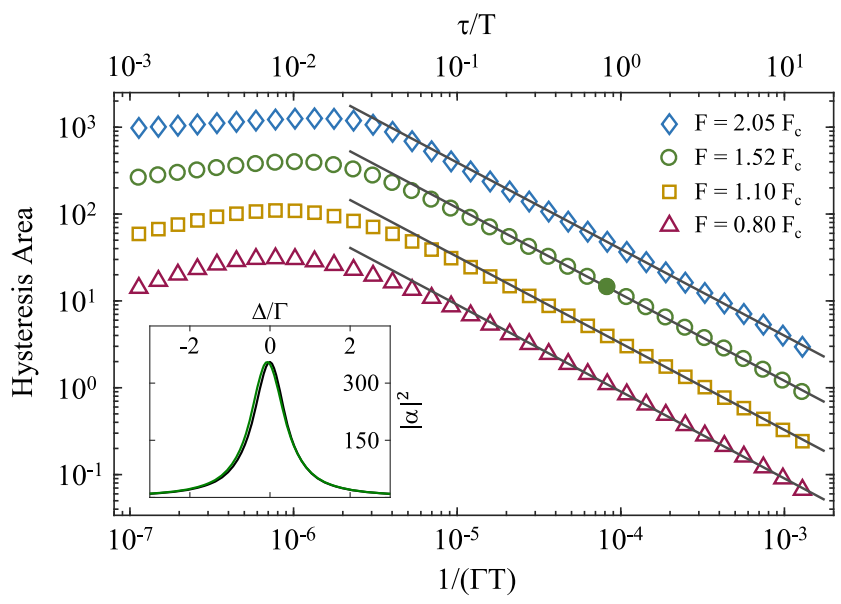

FIG. 4. Calculated hysteresis area versus the scanning period $T$. Each symbol corresponds to a different driving amplitude $F$ relative to the critical amplitude needed for bistability $F_{c}$. Gray lines are power law with exponent -1 . Inset: Intracavity photon number $|\alpha|^{2}$ versus $\Delta / \Gamma$ for the period indicated by the filled circle in the main panel.
Fig. 4 over an extended range. As in experiments, we observe the onset of the -1 power law within the restricted speed range in Fig. 3(b).

Recent calculations [43] and experiments [13] on hysteretic cavities with instantaneous interactions observed a universal scaling of $A$ at low speeds due to quantum fluctuations. Coincidentally, the scaling exponent found in Refs. $[13,43]$ is also -1 , as in the present work. However, the scaling behavior here reported has an entirely different origin (i.e., due to noninstantaneous interactions and unrelated to fluctuations) and arises in the opposite regime of fast scans.

Next, we demonstrate a new regime of non-Markovian dynamics emerging from the interplay of noninstantaneous effective photon-photon interactions and white noise in the laser amplitude and phase. For fixed $F$ and $\Delta / \Gamma$ within the bistability, $|\alpha|^{2}$ randomly switches between metastable states as shown in Supplemental Material [46]. Based on many long trajectories $|\alpha(t)|^{2}$, we calculate residence time distributions (RTDs) in the metastable states. For example, Figs. 5(a) and 5(b) show RTDs in the lower metastable state for two different $\tau$. For $\tau \ll \Gamma^{-1}$, the dynamics is Markovian, and RTDs decay exponentially as in Fig. 5(a). For $\tau>\Gamma^{-1}$, RTDs become increasingly peaked with increasing $\tau$. The deviation from exponential decay [see the unfitting line in Fig. 5(b)] evidences non-Markovian dynamics [56]. NonMarkovian dynamics are usually associated with noninstantaneous system-environment interactions (dissipation) and with colored noise in the driving force according to the fluctuation-dissipation relation [53,54,57-60]. In contrast, we demonstrate non-Markovian dynamics emerging from noninstantaneous effective photon-photon interactions, which suppress fast switchings between metastable states regardless of the noise spectrum. Experimentally, our system also experiences colored noise in $\Delta$ due to mechanical fluctuations of our mirrors with a high-frequency cutoff. In Supplemental Material, we show how this noise influences single-shot dynamic hysteresis measurements [46].

Finally, we estimate the experimental thermal relaxation time by comparing hysteresis cycles in Figs. 2(a) and 2(b). Since in theory we set $\tau$ and the scanning speed for $\Delta / \Gamma$, we can convert $\tau$ to a range of $\Delta / \Gamma$ and vice versa. In Fig. 2, we
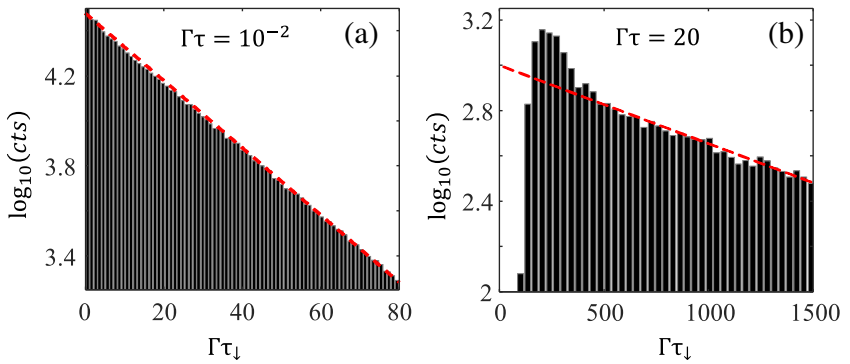

FIG. 5. Residence time distributions in the lower metastable state for different $\tau$. Dashed lines are exponential fits. The peaked distribution in (b) is indicative of non-Markovian dynamics. 
indicate the $\Delta / \Gamma$ range corresponding to $\tau$ by dashed lines. As expected, the $\Delta / \Gamma$ range corresponding to $\tau$ increases with the speed. For the lowest speed $f, T_{b} \gg \tau$ and the overshoot observed when $\Delta$ decreases is the main feature unanticipated by the standard Kerr model. For $7 f, T_{b} \sim \tau$ and the overshoot is almost as wide as the bistability. For $49 f, T_{b}<\tau$ and we have a close-to-linear response. Experiments in Fig. 2(a) display similar behavior as calculations in Fig. 2(b). Hence, in the same fashion, we indicate the $\Delta / \Gamma$ range corresponding to $\tau$ by dashed lines in Fig. 2(a). Based on this range of $\Delta / \Gamma$ and our knowledge of the experimental scanning speed, all three measurements in Fig. 2(a) are consistent with a relaxation time $\tau=16 \pm 1 \mu \mathrm{s}$.

In summary, we demonstrated signatures of scaling, universality, and non-Markovianity, in the dynamics of a laser-driven cavity with thermo-optical nonlinearity. Because of noninstantaneous effective photon-photon interactions, the optical hysteresis area is maximized at a finite scanning speed. At high speeds, we discovered a universal scaling behavior through which the hysteresis characterizing first-order phase transitions vanishes. Our findings suggest that oil-filled cavities are promising for exploring critical phenomena and new universality classes in systems with memory $[61,62]$. For slow or fixed driving conditions, our system exhibits peaked RTDs and nonMarkovian dynamic hysteresis. This suggests that our system could be used to test theorems of nonequilibrium fluctuations [63], entropy production [64], and speed limits [65] in non-Markovian regimes. Beyond single-cavity physics, our observation of optical bistability in oil-filled cavities paves the way for realizing bistable coupled cavities [66] and cavity arrays at room temperature. Such arrays could be used to probe Ising-type phase transitions [10] or to solve combinatorial optimization problems [22-24].

This work is part of the research program of the Netherlands Organisation for Scientific Research (NWO). We thank Ricardo Struik and Niels Commandeur for technical support and Ewold Verhagen and Femius Koenderink for stimulating discussions. S. R. K. R. acknowledges a NWO Veni grant with file number 016.Veni.189.039.

*s.rodriguez@amolf.nl

[1] R. Graham and H. Haken, Laserlight-First example of a second-order phase transition far away from thermal equilibrium, Z. Phys. 237, 31 (1970).

[2] R. Roy and L. Mandel, Optical bistability and first order phase transition in a ring dye laser, Opt. Commun. 34, 133 (1980).

[3] M. O. Scully, Condensation of $N$ Bosons and the Laser Phase Transition Analogy, Phys. Rev. Lett. 82, 3927 (1999).

[4] A. Le Boité, G. Orso, and C. Ciuti, Steady-State Phases and Tunneling-Induced Instabilities in the Driven Dissipative Bose-Hubbard Model, Phys. Rev. Lett. 110, 233601 (2013).
[5] H. J. Carmichael, Breakdown of Photon Blockade: A Dissipative Quantum Phase Transition in Zero Dimensions, Phys. Rev. X 5, 031028 (2015).

[6] J. J. Mendoza-Arenas, S. R. Clark, S. Felicetti, G. Romero, E. Solano, D. G. Angelakis, and D. Jaksch, Beyond meanfield bistability in driven-dissipative lattices: Bunchingantibunching transition and quantum simulation, Phys. Rev. A 93, 023821 (2016).

[7] R. M. Wilson, K. W. Mahmud, A. Hu, A. V. Gorshkov, M. Hafezi, and M. Foss-Feig, Collective phases of strongly interacting cavity photons, Phys. Rev. A 94, 033801 (2016).

[8] M. Fitzpatrick, N. M. Sundaresan, A. C. Y. Li, J. Koch, and A. A. Houck, Observation of a Dissipative Phase Transition in a One-Dimensional Circuit QED Lattice, Phys. Rev. X 7, 011016 (2017).

[9] J. M. Fink, A. Dombi, A. Vukics, A. Wallraff, and P. Domokos, Observation of the Photon-Blockade Breakdown Phase Transition, Phys. Rev. X 7, 011012 (2017).

[10] M. Foss-Feig, P. Niroula, J. T. Young, M. Hafezi, A. V. Gorshkov, R. M. Wilson, and M. F. Maghrebi, Emergent equilibrium in many-body optical bistability, Phys. Rev. A 95, 043826 (2017).

[11] W. Casteels, R. Fazio, and C. Ciuti, Critical dynamical properties of a first-order dissipative phase transition, Phys. Rev. A 95, 012128 (2017).

[12] M. Biondi, G. Blatter, H. E. Türeci, and S. Schmidt, Nonequilibrium gas-liquid transition in the driven-dissipative photonic lattice, Phys. Rev. A 96, 043809 (2017).

[13] S. R. K. Rodriguez, W. Casteels, F. Storme, N. Carlon Zambon, I. Sagnes, L. Le Gratiet, E. Galopin, A. Lemaître, A. Amo, C. Ciuti, and J. Bloch, Probing a Dissipative Phase Transition Via Dynamical Optical Hysteresis, Phys. Rev. Lett. 118, 247402 (2017).

[14] A. Angerer, S. Putz, D. O. Krimer, T. Astner, M. Zens, R. Glattauer, K. Streltsov, W. J. Munro, K. Nemoto, S. Rotter, J. Schmiedmayer, and J. Majer, Ultralong relaxation times in bistable hybrid quantum systems, Sci. Adv. 3, e1701626 (2017).

[15] T. Fink, A. Schade, S. Höfling, C. Schneider, and A. Imamoglu, Signatures of a dissipative phase transition in photon correlation measurements, Nat. Phys. 14, 365 (2018).

[16] F. Vicentini, F. Minganti, R. Rota, G. Orso, and C. Ciuti, Critical slowing down in driven-dissipative Bose-Hubbard lattices, Phys. Rev. A 97, 013853 (2018).

[17] D. O. Krimer and M. Pletyukhov, Few-Mode Geometric Description of a Driven-Dissipative Phase Transition in an Open Quantum System, Phys. Rev. Lett. 123, 110604 (2019).

[18] S. Finazzi, A. Le Boité, F. Storme, A. Baksic, and C. Ciuti, Corner-Space Renormalization Method for DrivenDissipative Two-Dimensional Correlated Systems, Phys. Rev. Lett. 115, 080604 (2015).

[19] L. M. Sieberer, M. Buchhold, and S. Diehl, Keldysh field theory for driven open quantum systems, Rep. Prog. Phys. 79, 096001 (2016).

[20] M. J. Hartmann, Quantum simulation with interacting photons, J. Opt. 18, 104005 (2016).

[21] C. Noh and D. G. Angelakis, Quantum simulations and many-body physics with light, Rep. Prog. Phys. 80, 016401 (2016). 
[22] T. Leleu, Y. Yamamoto, S. Utsunomiya, and K. Aihara, Combinatorial optimization using dynamical phase transitions in driven-dissipative systems, Phys. Rev. E 95, 022118 (2017).

[23] T. Leleu, Y. Yamamoto, P. L. McMahon, and K. Aihara, Destabilization of Local Minima in Analog Spin Systems by Correction of Amplitude Heterogeneity, Phys. Rev. Lett. 122, 040607 (2019).

[24] O. Kyriienko, H. Sigurdsson, and T. C. H. Liew, Probabilistic solving of $n p$-hard problems with bistable nonlinear optical networks, Phys. Rev. B 99, 195301 (2019).

[25] A. Opala, S. Ghosh, T. C. H. Liew, and M. Matuszewski, Neuromorphic Computing in Ginzburg-Landau PolaritonLattice Systems, Phys. Rev. Applied 11, 064029 (2019).

[26] I. Carusotto and C. Ciuti, Quantum fluids of light, Rev. Mod. Phys. 85, 299 (2013).

[27] P. D. Drummond and D. F. Walls, Quantum theory of optical bistability. I. Nonlinear polarisability model, J. Phys. A 13, 725 (1980).

[28] E. P. Gross, Structure of a quantized vortex in boson systems, Nuovo Cimento (1955-1965) 20, 454 (1961).

[29] L. Pitaevskii, Vortex lines in an imperfect Bose gas, Sov. Phys. JETP 13, 451 (1961).

[30] F. Dalfovo, S. Giorgini, L. P. Pitaevskii, and S. Stringari, Theory of Bose-Einstein condensation in trapped gases, Rev. Mod. Phys. 71, 463 (1999).

[31] V. L. Ginzburg and L. D. Landau, On the theory of superconductivity, in On Superconductivity and Superfluidity: A Scientific Autobiography (Springer, Berlin, Heidelberg, 1950), pp. 113-137, https://doi.org/10.1007/978-3-54068008-6_4.

[32] L. A. Lugiato and R. Lefever, Spatial Dissipative Structures in Passive Optical Systems, Phys. Rev. Lett. 58, 2209 (1987).

[33] J. Goldstone, Field theories with "superconductor" solutions, Nuovo Cimento 19, 154 (1961).

[34] V. R. Almeida and M. Lipson, Optical bistability on a silicon chip, Opt. Lett. 29, 2387 (2004).

[35] T. Carmon, L. Yang, and K. J. Vahala, Dynamical thermal behavior and thermal self-stability of microcavities, Opt. Express 12, 4742 (2004).

[36] M. Notomi, A. Shinya, S. Mitsugi, G. Kira, E. Kuramochi, and T. Tanabe, Optical bistable switching action of Si highQ photonic-crystal nanocavities, Opt. Express 13, 2678 (2005).

[37] G. Priem, P. Dumon, W. Bogaerts, D. V. Thourhout, G. Morthier, and R. Baets, Optical bistability and pulsating behaviour in silicon-on-insulator ring resonator structures, Opt. Express 13, 9623 (2005).

[38] Y. Shi, X. Chen, F. Lou, Y. Chen, M. Yan, L. Wosinski, and M. Qiu, All-optical switching of silicon disk resonator based on photothermal effect in metal-insulator-metal absorber, Opt. Lett. 39, 4431 (2014).

[39] M. Brunstein, R. Braive, R. Hostein, A. Beveratos, I. Robert-Philip, I. Sagnes, T. J. Karle, A. M. Yacomotti, J. A. Levenson, V. Moreau, G. Tessier, and Y. D. Wilde, Thermo-optical dynamics in an optically pumped photonic crystal nano-cavity, Opt. Express 17, 17118 (2009).

[40] M. Sodagar, M. Miri, A. A. Eftekhar, and A. Adibi, Optical bistability in a one-dimensional photonic crystal resonator using a reverse-biased pn-junction, Opt. Express 23, 2676 (2015).

[41] K. P. Kalinin and N. G. Berloff, Simulating Ising and $n$-State Planar Potts Models and External Fields with Nonequilibrium Condensates, Phys. Rev. Lett. 121, 235302 (2018).

[42] P. Jung, G. Gray, R. Roy, and P. Mandel, Scaling Law for Dynamical Hysteresis, Phys. Rev. Lett. 65, 1873 (1990).

[43] W. Casteels, F. Storme, A. Le Boité, and C. Ciuti, Power laws in the dynamic hysteresis of quantum nonlinear photonic resonators, Phys. Rev. A 93, 033824 (2016).

[44] L. Pickup, K. Kalinin, A. Askitopoulos, Z. Hatzopoulos, P. G. Savvidis, N. G. Berloff, and P. G. Lagoudakis, Optical Bistability Under Nonresonant Excitation in Spinor Polariton Condensates, Phys. Rev. Lett. 120, 225301 (2018).

[45] A. A. P. Trichet, P. R. Dolan, D. M. Coles, G. M. Hughes, and J. M. Smith, Topographic control of open-access microcavities at the nanometer scale, Opt. Express 23, 17205 (2015).

[46] See Supplemental Material at http://link.aps.org/ supplemental/10.1103/PhysRevLett.124.153603 for details about the experimental setup, calculations, and an estimate of the temperature rise in our bistable oil-filled cavity.

[47] A. Dreischuh, D. N. Neshev, D. E. Petersen, O. Bang, and W. Krolikowski, Observation of Attraction Between Dark Solitons, Phys. Rev. Lett. 96, 043901 (2006).

[48] R. F. Souza, M. A. Alencar, M. R. Meneghetti, and J. M. Hickmann, Large nonlocal nonlinear optical response of castor oil, Opt. Mater. 31, 1591 (2009).

[49] A. García, S. Valbuena, R. Sarmiento, and F. Racedo, Measurement of the nonlinear optical properties of olive oil using z-scan, Opt. Pura Aplicada 48, 55 (2015).

[50] H. Abbaspour, G. Sallen, S. Trebaol, F. Morier-Genoud, M. T. Portella-Oberli, and B. Deveaud, Effect of a noisy driving field on a bistable polariton system, Phys. Rev. B 92, 165303 (2015).

[51] S. A. Khodier, Refractive index of standard oils as a function of wavelength and temperature, Opt. Laser Technol. 34, 125 (2002).

[52] S. R. K. Rodriguez, Enhancing the Speed and Sensitivity of a Nonlinear Optical Sensor with Noise, Phys. Rev. Applied 13, 024032 (2020).

[53] H. Mori, Transport, collective motion, and Brownian motion, Prog. Theor. Phys. 33, 423 (1965).

[54] P. Hänggi, Correlation functions and masterequations of generalized (non-Markovian) Langevin equations, Z. Phys. 31, 407 (1978).

[55] A. Hohl, H. J. C. van der Linden, R. Roy, G. Goldsztein, F. Broner, and S. H. Strogatz, Scaling Laws for Dynamical Hysteresis in a Multidimensional Laser System, Phys. Rev. Lett. 74, 2220 (1995).

[56] I. Goychuk and P. Hänggi, Non-Markovian Stochastic Resonance, Phys. Rev. Lett. 91, 070601 (2003).

[57] P. Hanggi and F. Mojtabai, Thermally activated escape rate in presence of long-time memory, Phys. Rev. A 26, 1168 (1982).

[58] A. V. Mokshin, R. M. Yulmetyev, and P. Hänggi, Simple Measure of Memory for Dynamical Processes Described by 
a Generalized Langevin Equation, Phys. Rev. Lett. 95, 200601 (2005).

[59] R. Farias, R. O. Ramos, and L. da Silva, Numerical solutions for non-Markovian stochastic equations of motion, Comput. Phys. Commun. 180, 574 (2009), special issue based on the Conference on Computational Physics 2008.

[60] R. L. S. Farias, R. O. Ramos, and L. A. da Silva, Stochastic Langevin equations: Markovian and non-Markovian dynamics, Phys. Rev. E 80, 031143 (2009).

[61] D. Nagy and P. Domokos, Nonequilibrium Quantum Criticality and Non-Markovian Environment: Critical Exponent of a Quantum Phase Transition, Phys. Rev. Lett. 115, 043601 (2015).

[62] J. Marino and S. Diehl, Driven Markovian Quantum Criticality, Phys. Rev. Lett. 116, 070407 (2016).
[63] T. Speck and U. Seifert, The Jarzynski relation, fluctuation theorems, and stochastic thermodynamics for non-markovian processes, J. Stat. Mech. (2007) L09002.

[64] A. Kutvonen, T. Ala-Nissila, and J. Pekola, Entropy production in a non-Markovian environment, Phys. Rev. E 92, 012107 (2015).

[65] S. Deffner and E. Lutz, Quantum Speed Limit for Non-Markovian Dynamics, Phys. Rev. Lett. 111, 010402 (2013).

[66] S. Dufferwiel, F. Li, A. A. P. Trichet, L. Giriunas, P. M. Walker, I. Farrer, D. A. Ritchie, J. M. Smith, M. S. Skolnick, and D. N. Krizhanovskii, Tunable polaritonic molecules in an open microcavity system, Appl. Phys. Lett. 107, 201106 (2015). 